\title{
Cerebrospinal fluid a synuclein concentrations in patients with positive $A D$ biomarkers and extrapyramidal symptoms
}

\author{
Izabela Winkel ${ }^{1,2} \cdot$ Natalia Ermann $^{3} \cdot$ Agnieszka Żelwetro $^{4} \cdot$ Bożydar Sambor $^{5} \cdot$ Barbara Mroczko $^{6,7}$. \\ Johannes Kornhuber ${ }^{3} \cdot$ Bogusław Paradowski $^{8} \cdot$ Piotr Lewczuk $^{3,6,7,9}$
}

Received: 9 March 2021 / Accepted: 7 May 2021 / Published online: 25 May 2021

(c) The Author(s) 2021

\begin{abstract}
Extrapyramidal symptoms (EP) are not uncommon in Alzheimer's Disease (AD); when present, they negatively influence the course of the disorder. A large proportion of AD patients shows concomitant Lewy bodies' pathology post mortem. Total $\alpha$ Synuclein ( $\alpha$ Syn) concentrations are frequently increased in the cerebrospinal fluid (CSF) of AD patients, but are decreased in Parkinson's Disease (PD) and Dementia with Lewy Bodies (DLB). $\alpha$ Syn CSF concentrations in AD patients with EP (EP+) have not been reported so far. $\alpha$ Syn and the four Neurochemical Dementia Diagnostics (NDD) CSF biomarkers, (A $\beta 1-42$, A $\beta 42 / 40$, Tau, and pTau181), interpreted according to the Erlangen Score algorithm, were measured in patients with positive NDD results and presence of extrapyramidal symptoms (NDD $+/ \mathrm{EP}+; n=26)$, in patients with positive NDD results and absence of extrapyramidal symptoms (NDD+/ EP-; $n=54$ ), and in subjects with negative NDD results (NDD-; $n=34$ ). Compared to the NDD - controls $(379.8 \pm 125.2 \mathrm{pg} / \mathrm{mL})$, NDD+ patients showed, on average, highly significantly increased CSF $\alpha$ Syn $(519 \pm 141.3 \mathrm{pg} / \mathrm{mL}, p<0.01)$, but without differences between NDD+ / EP+ and NDD+/ EP - subgroups $(p=0$. 38). Moderate but highly significant association was observed between concentrations of $\alpha$ Syn and Tau $(\mathrm{r}=0.47, p<0.01)$ and pTau $181(\mathrm{r}=0.65, p<0.01)$. Adjusted for diagnoses, age, and sex, subjects with more advanced neurodegeneration on neuroimaging showed significantly lower $\alpha$ Syn concentrations $(p<0.02)$. In the setting $\mathrm{AD}$ versus controls, the area under the receiver operating characteristic (ROC) curve was $0.804[0.712 ; 0.896]$ with the sensitivity and the specificity of 0.863 and 0.618 , respectively. $\alpha$ Syn in AD patients does not differentiate between subjects with- and without EP. Its increased average concentration reflects probably neurodegenerative process, and is not specific for any pathophysiologic mechanisms. Further studies are necessary to explain the role of CSF $\alpha$ Syn as a potential biomarker.
\end{abstract}

Keywords $\alpha$ Synuclein · Alzheimer's disease · Cerebrospinal fluid · Extrapyramidal symptoms $\cdot$ Biomarker

Abbreviations
$\begin{array}{ll}\alpha \operatorname{Syn} & \alpha \text { Synuclein } \\ \text { A } \beta 1-40 & \text { Amyloid } \beta \text { peptide with the amino acid } \\ & \text { sequence } 1 \text { to } 40\end{array}$

Piotr Lewczuk

Piotr.Lewczuk@uk-erlangen.de

1 Dementia Disorders Center of the Medical University of Wrocław, Ścinawa, Poland

2 Department and Clinic of Geriatrics, Medical University of Wrocław, Wrocław, Poland

3 Department of Psychiatry and Psychotherapy, Universitätsklinikum Erlangen, and Friedrich-Alexander Universität Erlangen-Nürnberg, Erlangen, Germany

4 Interdyscyplinarne Studia Doktoranckie Uniwersytetu SWPS, II Wydział Psychologii, Wrocław, Poland
A $\beta 1$-42 Amyloid $\beta$ peptide with the amino acid sequence 1 to 42

$\mathrm{A} \beta 42 / 40$ Concentration ratio of the peptides $A \beta 1-42$ and A $\beta 1-40$

AD Alzheimer Disease

5 Affidea Sp.Z O. O. Legnica, Legnica, Poland

6 Department of Neurodegeneration Diagnostics, Medical University of Białystok, Białystok, Poland

7 Department of Biochemical Diagnostics, University Hospital of Białystok, Białystok, Poland

8 Department of Neurology, Medical University of Wrocław, Wrocław, Poland

9 Department of Psychiatry and Psychotherapy, Lab for Clinical Neurochemistry and Neurochemical Dementia Diagnostics, Schwabachanlage 6, 91054 Erlangen, Germany 


$\begin{array}{ll}\text { AIC } & \text { Akaike's Information Criterion } \\ \text { AUC } & \text { Area Under the [ROC] Curve } \\ \text { CI } & \text { Confidence Interval } \\ \text { CSF } & \text { Cerebrospinal Fluid } \\ \text { DLB } & \text { Dementia with Lewy Bodies } \\ \text { EP } & \text { Extrapyramidal Symptoms } \\ \text { ES } & \text { Erlangen Score } \\ \text { LRT } & \text { Likelihood Ratio Test } \\ \text { M } & \text { A statistical model } \\ \text { MMSE } & \text { Mini Mental State Examination } \\ \text { MTA } & \text { Medial Temporal Lobe Atrophy } \\ \text { NDD } & \text { Neurochemical Dementia Diagnostics } \\ \text { NIA-AA } & \text { National Institute on Aging-Alzheimer's } \\ & \text { Disease Association } \\ \text { PD } & \text { Parkinson's Disease } \\ \text { pTau181 } & \text { Tau molecule phosphorylated at the amino acid } \\ & \text { position 181 } \\ \text { QC } & \text { Quality control } \\ \text { r } & \text { Pearson's Correlation Coefficient } \\ \text { ROC } & \text { Receiver Operating Characteristic } \\ \text { SD } & \text { Standard Deviation }\end{array}$

\section{Introduction}

In contrast to well established and generally accepted pattern of the cerebrospinal fluid (CSF) biomarkers for the diagnosis of Alzheimer's Disease (AD), which includes decreased values of $A \beta 1-42$ and $A \beta 42 / 40$, and increased concentrations of Tau and pTau181 (Lewczuk et al. 2018; Hansson et al. 2019), the diagnostic role of $\alpha$ Synuclein ( $\alpha$ Syn) in Parkinson's Disease (PD) and Dementia with Lewy Bodies (DLB) is still a matter of debate. There seems to be consensus in the literature that the total $\alpha$ Syn CSF concentrations are decreased in these synucleinopathies, compared to control subjects without neurodegeneration, but the acceptance of $\alpha \mathrm{Syn}$ as a routine biomarker is hampered by suboptimal diagnostic accuracy, relatively large inter-center variation, and lacking consensus which of the isomers should be targeted (Mollenhauer et al. 2008; Parnetti et al. 2016; Simonsen et al. 2016).

Growing body of literature shows co-occurrence of the neuropathologic findings characteristic for synucleinopathies in a large proportion of autopsy confirmed AD patients (Arai et al. 2001; Hamilton 2000; Slaets et al. 2013). This is often associated with spatial co-localization of $\alpha$ Syn and Tau lesions (Lippa et al. 1998; Marui et al. 2000). Furthermore, significantly increased levels of the intracellular $\alpha$ Syn were reported in the inferior temporal lobe of the brains of $\mathrm{AD}$ patients without detectable DLB pathology (Larson et al. 2017).

These observations, taken together with the postulated role of $\alpha$ Syn in pathophysiology of AD (Twohig and Nielsen
2019), lead to the obvious question, if CSF $\alpha$ Syn could be regarded as a potential biomarker, particularly in differential diagnosis of $\mathrm{AD}$ versus PD/DLB. Indeed, several studies addressing this issue have been published (Simonsen et al. 2016); (Twohig and Nielsen 2019), but little is known about $\alpha$ Syn CSF concentrations in subjects with AD and concomitant PD comorbidity. This seems to be particularly relevant, considering that majority of the published reports shows that, compared to the relevant control groups, $\alpha \mathrm{Syn}$ CSF concentrations are decreased in synucleinopathies but increased in AD.

Therefore, in this retrospective cross-sectional study we measured $\alpha$ Syn CSF concentrations in carefully selected AD patients whose clinical, neuroimaging, and neuropsychologic diagnoses were confirmed by the four core CSF AD biomarkers, and who either exhibited or did not exhibit extrapyramidal symptoms (EP). We contrasted the results with those of the control group consisting of patients without alterations in the core CSF AD biomarkers and without EP.

\section{Materials and methods}

\section{Patients and the CSF collection, neuroimaging, and the core CSF biomarkers}

The study was approved by the ethical committee at the Medical University of Wrocław, Poland. The patients were recruited at the Diagnostic and Research Center for Alzheimer's disease, Ścinawa, Poland. The AD diagnoses were performed according to the NIA-AA criteria (McKhann et al. 2011), which was, however, treated only as an additional, supportive criterion on the top of the CSF-based categorization. The CSF samples were collected by lumbar punctures into polypropylene test tubes. Portions of ca. $250 \mu \mathrm{L}$ for the NDD and $\alpha$ Syn analyses were immediately aliquoted and frozen at $-80{ }^{\circ} \mathrm{C}$. Aliquots were then shipped on dry ice to the Lab for Clinical Neurochemistry and Neurochemical Dementia Diagnostics, Erlangen, Germany, where the laboratory analyses were performed. In all cases, total protein concentration, glucose concentration, and cell count measured in the local laboratory. Samples contaminated with blood were not used for the study. In all subjects, the four core Neurochemical Dementia Diagnostics (NDD) CSF biomarkers were measured with ELISA: A $\beta 1-42, A \beta 1-40$ (IBL International, Hamburg, Germany), Tau, and pTau181 (Fujirebio Europe, Ghent, Belgium), according to the vendors' protocols. The results of the core biomarkers were interpreted according to the previously published and validated Erlangen Score (ES) algorithm (Lewczuk et al. 2009, 2015; Baldeiras et al. 2019; Skillback et al. 2019; Somers et al. 2019). The subjects with $\mathrm{ES} \geq 3$ were categorized as NDD+ patients $(n=80)$, and the subjects with $\mathrm{ES} \leq 1$ 
$(n=34)$ were categorized as NDD- controls. Furthermore, the NDD+ subjects were split into a subgroup with EP (NDD+/EP + $n=26$ ), including: bradykinesia (in 10 subjects), muscle rigidity with cogwheel or lead-pipe muscle rigidity (in 6 subjects), concurrence of both (in 3 subjects), and asymmetric tremor (in 7 subjects), and subgroup without $\mathrm{EP}(\mathrm{NDD}+/ \mathrm{EP}-n=54)$. Extrapyramidal symptoms did not cause pronounced motor and non-motor abnormalities and did not meet the criteria for atypical Parkinsonism. There were no episodes of psychotic productive symptoms in the study group. Neurologic conditions were assessed by two independent consultants experienced in neurogerontology.

In 101 subjects (70 NDD + patients and 31 NDD- controls), routine neuroimaging was performed with Signa HD XT 1.5 T scanner (GE Medical Healthcare, Chicago, IL, USA). For the evaluation of the structural alterations, Medial Temporal lobe Atrophy (MTA) scale was applied (Scheltens et al. 1992). The MTA-score is rated on the coronal T1-weighted images at a consistent slice position, in such a way that a slice through the corpus of the hippocampus is selected at the level of the anterior pons. The score is based on a visual rating of the width of the choroid fissure, the width of the temporal horn, and the height of the hippocampal formation. The score ranges from 0 (no atrophy) to 4 (severe volume loss of hippocampus). Results are agedependent: for a person younger than 75 years a score of 2 or more is abnormal. For older patients, a score of 3 or more is considered abnormal. A high MTA-score is a very sensitive indicator to diagnose Alzheimer's disease and is present in the vast majority of patients. In controls a positive score is almost always absent. The evaluation of the MTA was performed entirely independent of the CSF biomarkers, by an experienced neuroradiologist who did not have access to the CSF data.

\section{a Synuclein assay}

Concentrations of $\alpha$ Syn in the patients' CSF samples were assayed with a chemiluminescence method (Meso Scale Diagnostics; Rockville, MD, USA), according to the vendor's protocol. In preliminary experiments, four samples with different $\alpha$ Syn concentrations were pre-diluted with assay's buffer $1: 4,1: 8,1: 16$, and 1:32 to test for linearity and precision of the method. Resulting from this experiment, 1:8 dilution was chosen. All analyses were performed in duplicates. To test for inter-assay precision, a quality control (QC) sample of human pooled CSF was used.

\section{Statistical analysis}

The number of subjects for the study was estimated under the condition of Satterthwaite's test for the populations with non-equal variances, with between-groups differences approximated according to the data in the literature (Mackin et al. 2015); the statistical significance and the power were set to 0.05 and 0.9 , respectively. To avoid inconclusive results, in case we had obtained betweengroups differences lower than those in the literature, the number of the study subjects was increased to 114 .

If not stated otherwise, the results of the continuous variables are presented as averages \pm standard deviations (SD) or $95 \%$ confidence intervals $(95 \% \mathrm{CI})$. The results of the categorical variables are presented as percentages, and compared to one another with $\chi^{2}$ and/or Kruskal - Wallis test. Statistical analyses were used contrasting: (a) the total NDD+ group versus NDD- controls, with Helmert contrast, and (b) the NDD+ with $\mathrm{EP}(\mathrm{NDD}+/ \mathrm{EP}+)$ group versus NDD+ without EP (NDD+/ EP-) group. Correlations between continuous variables, unadjusted for other covariates, are presented as Pearson's correlation coefficients (r).

Associations between CSF $\alpha$ Syn concentrations and the disease categories, adjusted for explanatory variables, were analyzed with a series of linear regression models. First, a model with clinical- neurochemical categorization only was fitted (M0), subsequently supplemented with the demographic data (M1), supplemented further with the time of the disease duration and the MMSE test score (M2), and finally with the presence of the metabolic syndrome (diabetes mellitus type 2 and/or obesity) and the results of the MTA score (M3). If the numbers of the patients in the models were equal, the models were compared with the Likelihood Ratio test (LRT); otherwise the models were compared with the Akaike's Information Criterion (AIC). Taking into account the results of the models M0 - M3, in the final regression model (M4) age, sex, and MTA results were retained. Finally, interactions between the explanatory variables and the disease categorization variable were tested.

The $\alpha$ Syn concentration cut off, optimized for the separation of NDD- and NDD+ groups, was obtained at the maximized Youden index. The area under the receiver operating characteristic (ROC) curve (AUC) was calculated with nonparametric method. Logistic regression was used to model the sensitivity and the specificity as a function of covariates, according to Coughlin et al. (Coughlin et al. 1992), slightly modified by introducing an interaction term for covariatesby-disease status. The diagnostic accuracy was defined as the conditional probability of the agreement between the result of the $\alpha$ Syn test and the disease status, and was modelled, conditional on age, with logistic regression. If not stated otherwise, estimates in all models were obtained with the Maximum Likelihood method.

A $p<0.05$ was considered significant. All statistical analyses were performed with Stata 14.2 (StataCorp, College Station, TX, USA). 


\section{Results}

\section{Demographic, neuropsychologic, neuroimaging, and the core CSF biomarkers results}

Demographic and neuropsychologic data of the patients, and the concentrations of their core CSF biomarkers, are presented in Table 1. In one case of pTau181 and in three cases of Tau, unmeasurably low concentrations were obtained. For statistical analyses, these results were set to the lowest assays' standard concentrations $(15.6 \mathrm{pg} / \mathrm{mL}$ and $40 \mathrm{pg} / \mathrm{mL}$, respectively).

Briefly, NDD+ patients compared to the NDD- controls were on average significantly older, had expectedly lower concentrations of $A \beta 1-42$ and $A \beta 42 / 40$ ratio, higher Tau and pTau181 concentrations, and lower MMSE score. There were no statistically significant differences in A $\beta 1-40$ concentrations between the two groups. The distributions of neither gender $(p=0.14)$ nor presence of the metabolic syndrome $(p=0.22)$ differed significantly between the two groups. None of the metrics differed significantly between the NDD/EP + and the NDD/EP- patients.

The distribution of the MTA scores is presented in Table 2. A non-significant tendency towards higher scores in the NDD+ patients compared to the NDD- controls $(p=0.054)$ was observed.

\section{a Synuclein assay performance}

Four dilution factors $(1: 4,1: 8,1: 16$, and 1:32) tested in the preliminary experiments resulted in practically the same concentrations (after taking the multiplication factor into consideration), as well as neglectable differences of the intra-assay imprecision (data not shown), reconfirming the linearity and the precision of the method. Median of the ranges-to-averages of the duplicate measurements of the 114 patients CSF samples was $2.1 \%$, with inter-quartile range: $1.0-3.6 \%$; CV of the average results of the QC sample in
Table 2 Distribution of the MTA scores in the NDD+ patients and the NDD- controls. Presented are the numbers and the percentages of the total number per given group

\begin{tabular}{llllll}
\hline \multicolumn{5}{c}{ MTA score } \\
\cline { 2 - 6 } & 0 & 1 & 2 & 3 & 4 \\
\hline NDD- $(n=31)$ & $12(39 \%)$ & $12(39 \%)$ & $4(13 \%)$ & $3(9 \%)$ & $0(0 \%)$ \\
NDD+ $(n=70)$ & $11(16 \%)$ & $26(37 \%)$ & $19(27 \%)$ & $9(13 \%)$ & $5(7 \%)$ \\
\hline
\end{tabular}

the four analytical runs (i. e. the inter-assay imprecision) was $7.6 \%$.

\section{a Synuclein in the CSF; correlation with the core CSF biomarkers}

We observed statistically highly significant difference between unadjusted average $\alpha$ Syn CSF concentrations in NDD+ patients and the NDD- controls $(p<0.01)$. We did not observe statistically significant difference between unadjusted $\alpha$ Syn CSF concentrations in NDD+ / EP+ and NDD+ / EP- patients ( $p=0.38$; Fig. 1$). \alpha$ Syn correlated moderately but significantly with age $(\mathrm{r}=0.29, p<0.01)$, $\mathrm{A} \beta 1-40(\mathrm{r}=0.42, p<0.01), \mathrm{A} \beta 42 / 40(\mathrm{r}=-0.38, p<0.01)$, Tau $(\mathrm{r}=0.47, p<0.01)$, and pTau181 $(\mathrm{r}=0.65, p<0.01)$, but not with $\mathrm{A} \beta 1-42(\mathrm{r}=-0.16, p=0.09)$.

In the regression models M0-M3 (Table 3), we observed significant association of the CSF $\alpha$ Syn concentrations with patients categorization, age, sex, and the MTA score, adjusted for the covariates as indicated. We did not observe association of the $\alpha$ Syn CSF concentrations with the duration of the disease, the MMSE score, and presence of the metabolic syndrome. Hence, in the final model (M4) age, sex, and the MTA sore were retained. In this model, holding other covariates constant, NDD+ patients had significantly higher average $\alpha$ Syn concentrations than the NDD- controls $(\beta=114.2, p<0.01)$, but there were no significant difference between NDD+ / EP + and NDD+/ EP- patients $(\beta=-55.0, p=0.11)$. Adjusted for other covariates, female
Table 1 Age, CSF biomarkers, and the MMSE score of the patients. Presented are averages and standard deviations

\begin{tabular}{lllll}
\hline & $\begin{array}{l}\text { NDD- } \\
n=34\end{array}$ & $\begin{array}{l}\text { NDD+ } \\
n=80\end{array}$ & $\begin{array}{l}\text { NDD+/EP- } \\
n=54\end{array}$ & $\begin{array}{l}\text { NDD+/EP+ } \\
n=26\end{array}$ \\
\hline Age (yrs.) & $64.5 \pm 7.5$ & $70.6 \pm 8.6^{*}$ & $70.4 \pm 8.2$ & $71.0 \pm 9.6$ \\
A $\beta 1-40$ & $15,320 \pm 4380$ & $16,250 \pm 5170$ & $16,880 \pm 4900$ & $14,930 \pm 5560$ \\
A $\beta 1-42$ & $1021 \pm 277$ & $510.6 \pm 135.7^{*}$ & $530.4 \pm 138.7$ & $469.5 \pm 121.5$ \\
A $\beta 42 / 40$ & $0.069 \pm 0.018$ & $0.033 \pm 0.009^{*}$ & $0.033 \pm 0.008$ & $0.034 \pm 0.011$ \\
Tau & $204 \pm 70.2$ & $661 \pm 322^{*}$ & $686 \pm 366$ & $608 \pm 197$ \\
pTau181 & $42.5 \pm 11.0$ & $93.9 \pm 23.2^{*}$ & $95.6 \pm 23.4$ & $90.4 \pm 22.8$ \\
$\alpha$ Synuclein & $379.8 \pm 125.2$ & $519 \pm 141.3^{*}$ & $528.4 \pm 151.0$ & $499.5 \pm 119.2$ \\
MMSE & $24.7 \pm 4.8$ & $19.1 \pm 5.1^{*}$ & $19.3 \pm 5.3$ & $18.8 \pm 4.7$ \\
\hline
\end{tabular}

*significantly different $(p<0.05)$ compared to the NDD- control group 


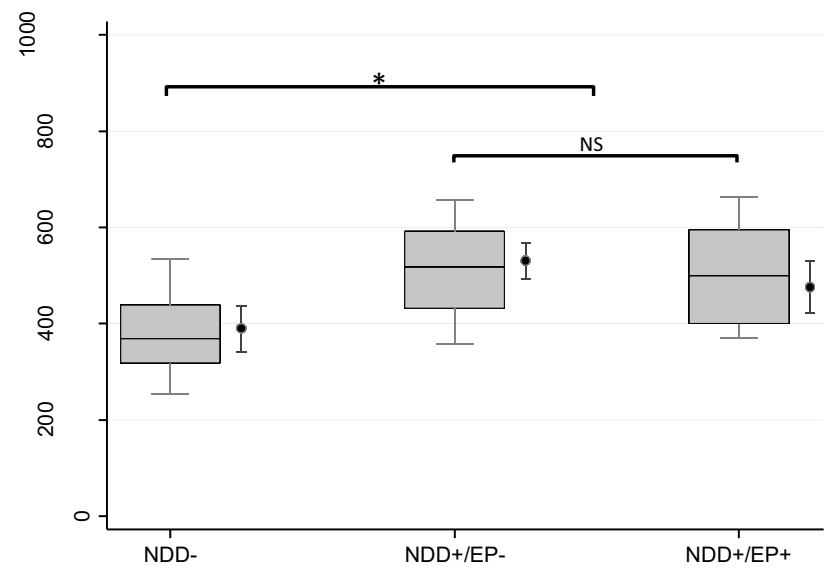

Fig. $1 \alpha$ Syn CSF concentrations in the three groups. Presented are: unadjusted medians (horizontal lines), 25-75 percentiles (boxes), and 10-90 percentiles (box whiskers). Right to the boxplots, adjusted linear marginal predictors (dots) with the corresponding $95 \%$ confidence intervals (dot whiskers) are presented post-estimated after the final regression model (M4)

gender $(\beta=-85.8, p<0.01)$, age $(\beta=5.43, p<0.01)$, and MTA score $(p<0.02)$ were significantly associated with $\alpha$ Syn concentrations. Interestingly, keeping other covariates constant, patients with the MTA sore $3(\beta=-132.9$, $p=0.012)$, and the MTA score $4(\beta=-158.4, p=0.03)$ had lower average $\alpha$ Syn concentrations than those with the MTA score 0 . We did not observe such association between higher
MTA scores and alterations of the concentration of Tau, conditional on other covariates (data not shown). Regression coefficients from this model are presented in Fig. 2, and marginal predictions of the CSF $\alpha$ Syn concentrations resulting from this model are also presented in Fig. 1. We did not observe significant interactions between either age or sex and the NDD categorization of the patients in this model.

\section{Sensitivity, specificity, and accuracy of a Synuclein as a potential AD biomarker}

ROC curve is presented in Fig. 3. The AUC was 0.804 [0.712; 0.896]. At the Youden point, maximizing the sum of the diagnostic sensitivity and the specificity separating the NDD+ patients from the NDD- controls, the cut off value of the $\alpha$ Syn concentration was $383 \mathrm{pg} / \mathrm{mL}$. The sensitivity, the specificity, and the accuracy were 0.863 [0.787; 0.938], 0.618 [0.454; 0.781$]$, and 0.798 [0.715; 0.864], respectively. In the logistic models, we did not find significant association of demographic data with the diagnostic metrics of $\alpha$ Syn (data not shown).

\section{Discussion}

In the current study, we observed increased concentrations of $\alpha$ Syn in the CSF of patients with AD, whose diagnoses were supported by pathologic CSF biomarkers and neuroimaging

Table 3 Regression models testing the associations of the variables

\begin{tabular}{|c|c|c|c|c|c|}
\hline \multirow[t]{3}{*}{ Explanatory variable } & \multicolumn{5}{|l|}{ Regression models } \\
\hline & M0 & M1 & M2 & M3 & M4 \\
\hline & $n=114$ & $n=114$ & $n=113$ & $n=113$ & $n=101$ \\
\hline \multicolumn{6}{|c|}{ Clinical-neurochemical categories ${ }^{1}$, compared to the reference category (NDD-): } \\
\hline $\mathrm{NDD}+/ \mathrm{EP}-$ & $148.6[89.3 ; 208.0]^{*}$ & $141.4[80.7 ; 202.1]^{*}$ & $136.4[68.0 ; 204.7]^{*}$ & $141.5[69.6 ; 213.4]^{*}$ & $141.7[78.0 ; 205.4]^{*}$ \\
\hline $\mathrm{NDD}+/ \mathrm{EP}+$ & $119.8[49.1 ; 190.4]^{*}$ & $97.6[26.4 ; 168.8]^{*}$ & $92.1[13.2 ; 171.1]^{*}$ & $87.5[3.9 ; 171.1]^{*}$ & $86.7[14.1 ; 159.3]^{*}$ \\
\hline Age (yrs.) & & $3.5[0.5 ; 6.5]^{*}$ & $3.5[0.4 ; 6.6]^{*}$ & $5.4[1.5 ; 9.3]^{*}$ & $5.4[1.7 ; 9.2]^{*}$ \\
\hline Female gender & & $-62.7[-115.4 ;-9.9]^{*}$ & $\begin{array}{l}-65.4[-119.8 \\
-11.1]^{*}\end{array}$ & $\begin{array}{l}-86.5[-143.8 \\
-29.1]^{*}\end{array}$ & $-85.8[-140.9 ;-30.6]^{*}$ \\
\hline MMSE & & & $-0.8[-6.0 ; 4.4]$ & $0.1[-5.4 ; 5.6]$ & \\
\hline Disease durat. (yrs.) & & & $-3.2[-13.9 ; 7.4]$ & $-1.2[-12.2 ; 9.8]$ & \\
\hline Metabolic synd & & & & $-2.8[-58.0 ; 52.3]$ & \\
\hline \multicolumn{6}{|c|}{ MTA score ${ }^{1}$, compared to the reference category $(\mathrm{MTA}=0)$ : } \\
\hline 1 & & & & $-18.0[-91.4 ; 55.4]$ & $-19.3[-89.4 ; 50.8]$ \\
\hline 2 & & & & $-0.5[-88.6 ; 87.6]$ & $-1.4[-86.6 ; 83.7]$ \\
\hline 3 & & & & $\begin{array}{l}-131.6[-240.1 \\
-23.1]^{*}\end{array}$ & $\begin{array}{l}-132.9[-236.2 \\
-29.6]^{*}\end{array}$ \\
\hline 4 & & & & $\begin{array}{l}-154.5[-304.1 \\
-5.0]^{*}\end{array}$ & $\begin{array}{l}-158.4[-300.1 \\
-16.7]^{*}\end{array}$ \\
\hline AIC & 1448 & 1443 & 1435 & 1268 & 1274 \\
\hline
\end{tabular}

${ }^{1}$ Coefficients of all categories are jointly significantly different from the corresponding reference category (Wald test) 


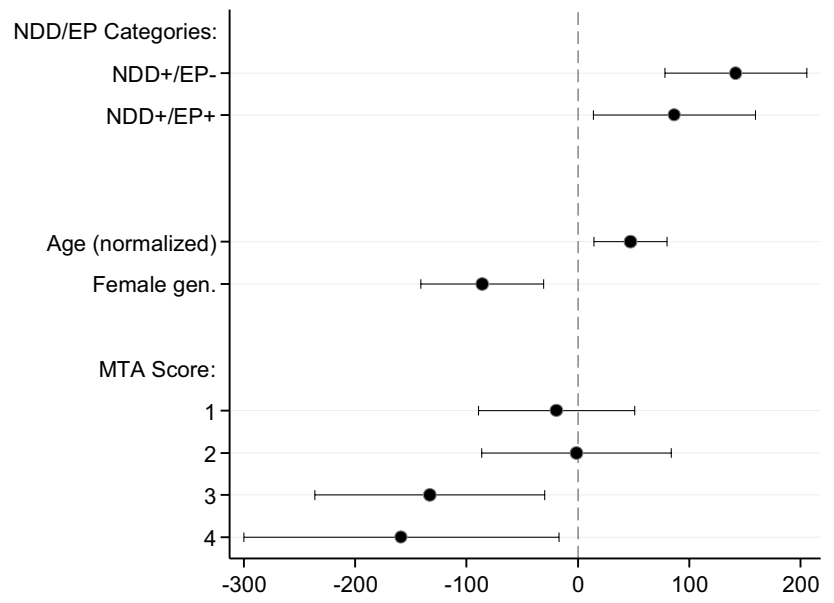

Fig. 2 Regression coefficients (dots) and their 95\% confidence intervals (spikes) from the final regression model (M4). For better visualization of the coefficient and CIs of age, the variable was normalized (divided by its standard deviation)

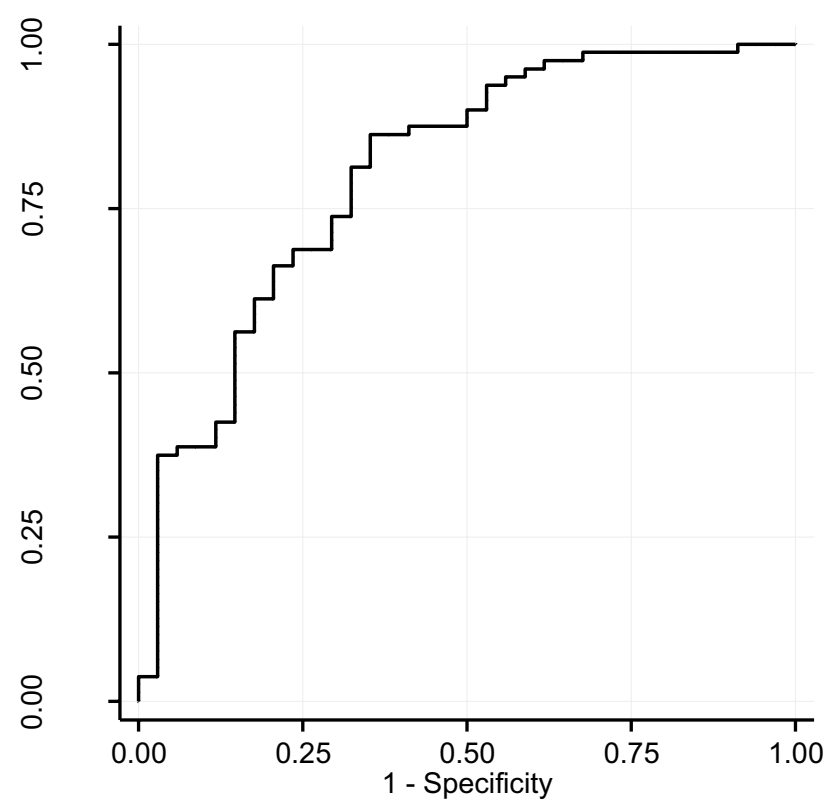

Fig. 3 ROC curve in the setting NDD+ versus NDD-

findings, compared to the non-demented controls without alterations of the CSF AD biomarkers. We did not observe differences between the average CSF $\alpha$ Syn concentrations of AD patients with- and without EP. Weak, though significant, association between CSF $\alpha$ Syn and A $\beta 1-40$ and $A \beta 42 / 40$ was observed, with somehow stronger association with Tau and pTau 181 .

EPs are not uncommon in AD, with as much as $22.6 \%$ of community-dwelling $\mathrm{AD}$ patients presenting with them in the course of the disease (Portet et al. 2009). The frequency and severity of EP appear to increase over time along with
AD severity (Ellis et al. 1996). Their clinical significance is poorly understood, and they probably result from different underlying mechanisms, and location or type of lesions, but clear association exists between presence and intensity of EP in AD subjects and more severe cognitive impairment, rapid cognitive decline, higher probability of institutionalization and higher socioeconomic burden (Portet et al. 2009).

A vast majority of studies published so far reports decreased CSF concentrations of total $\alpha$ Syn in PD subjects, compared to both healthy controls and/or patients with other neuropsychiatric disorders (a meta-analysis in (Eusebi et al. 2016)). On the other hand, large proportion of papers shows that CSF total $\alpha$ Syn concentrations tend to increase in AD compared to the controls (Twohig and Nielsen 2019). Increased $\alpha$ Syn concentrations in the AD subjects, along with moderate correlation between $\alpha$ Syn and Tau and/or pTau 181 in the current study reconfirm the data and the conclusions reported by many investigators, that the increased CSF $\alpha$ Syn in AD seems to rather reflect unspecific neurodegeneration and not specific processes characteristic for AD (Oeckl et al. 2016; Korff et al. 2013; Slaets et al. 2014; Majbour et al. 2017). For example, Slaets et al. found a significant, moderately positive correlation between Tau and $\alpha$ Syn and between pTau181 and $\alpha$ Syn in AD patients but not in DLB patients (Slaets et al. 2014) Interestingly, not only association of $\alpha$ Syn with biomarkers of neurodegeneration (Tau) but also with biomarkers more specific for AD (A $\beta$ peptides) was postulated; Buddhala et al. observed a positive correlation between CSF $\alpha$ Syn and A $\beta 1-42$ in PD patients, but not in controls, confirming a pathophysiologic connection between the metabolisms of these proteins in PD (Buddhala et al. 2015). Taken together, considering that predominant source of $\alpha$ Syn in the brain are presynaptic neuronal terminals, it seems reasonable to hypothesize that degenerating neurons passively release $\alpha$ Syn molecules, which then diffuse to the CSF at increased rate.

Our cohorts were selected in such a way, that the results of the four AD CSF biomarkers (A $\beta 1-42, A \beta 42 / 40$ ratio, Tau, and pTau181) were considered the major categorization criterion, with the patterns of the CSF biomarkers interpreted according to the Erlangen Score algorithm (Lewczuk et al. 2009, 2015). We believe that the CSF biomarkers, once established, properly validated, and consequently controlled for quality, are the most objective, investigator-independent diagnostic tools, resistant to all kinds of bias linked to an investigators (perhaps suboptimal) experience. This approach, taken together with moderate correlation between $\alpha$ Syn and Tau and/or pTau181, might explain why $\alpha$ Syn sensitivity and specificity, in the setting AD versus controls, resulted in our study in somehow larger ROC AUC and better diagnostic accuracy than those metrics reported by (Korff et al. 2013), and comparable to those reported by (Majbour et al. 2017). We believe that in a study like ours, with CSF 
biomarkers treated as an important categorization criterion, good performance of $\alpha \mathrm{Syn}$ as a potential biomarker is rather a side effect of its correlation with the biomarkers of neurodegeneration (Tau and pTau181) and not its individual characteristic. Therefore, the results of the ROC analysis we are reporting in this study must be treated exclusively as an additional statistics, perhaps helpful in comparison our results to those reported by others, and not as a claim that $\alpha$ Syn could be of use as a specific biomarker.

We did not observe difference in the average CSF concentrations of $\alpha$ Syn between AD patients with $\mathrm{EP}$ and $\mathrm{AD}$ patients without them. Since neuropathologic findings characteristic for $\mathrm{AD}$ and $\mathrm{PD} / \mathrm{DLB}$ overlap in a large proportion of subjects diagnosed post mortem and the literature shows clear tendencies towards increased $\alpha$ Syn in AD versus decreased $\alpha$ Syn in PD/DLB, a question raises whether patients presenting with dementia due to $\mathrm{AD}$ and concomitant clinical PD symptoms would show the net increase, decrease, or perhaps lack of alterations of the CSF $\alpha$ Syn. Toledo et al. found hallucinations as strong predictor of coincident $\alpha$ Syn aggregation in AD patients in an autopsycontrolled study (Toledo et al. 2013). Similarly, Mackin et al. found decreased CSF $\alpha$ Syn in AD patients with hallucinations and, when adjusted for Tau concentrations, with longitudinal decline in performance of executive function, $i$. $e$. two clinical symptoms often observed in DLB, compared to the AD patients without these symptoms. They concluded that reduction in $\alpha$ Syn concentration in these subjects may represent an association between AD and DLB-like symptomatology (Mackin et al. 2015). On the other hand, however, they also found reduced $\alpha$ Syn concentrations in AD patients with accelerated decline of memory and language, which is difficult to explain, as these domains are not characteristic for DLB. Therefore, we believe that explanations other than Lewy bodies' pathology for the presence of parkinsonism, such as EP, in AD must be considered. Such underlying mechanisms may include the presence of senile plaques in the putamen, caudate and substantia nigra, and the presence of neurofibrillary tangles in the substantia nigra (Liu et al. 1997; Burns et al. 2005).

Perhaps the most unexpected finding of our study is the decreasing average concentrations of $\alpha$ Syn, but not Tau, with advancing neurodegeneration process observed in neuroimaging (expressed as the MTA score), conditional on the diagnoses, age, and sex of the subjects. A simple explanation that more intense neuronal loss leaves behind less neurons capable to act as a source of $\alpha$ Syn is probably oversimplification, as such association was not observed for Tau. Hence, it might be speculated that the (passive) release of $\alpha$ Syn and Tau from degenerating neurons follows different patterns. Interestingly, Mackin et al., following their findings of the reduced $\alpha$ Syn CSF concentrations in AD patients with accelerated overall cognitive deterioration, also concluded that the CSF $\alpha$ Syn may be reduced as an effect of an overall neuronal or synaptic loss (Mackin et al. 2015).

Our study is not without limitations. The most serious one is probably lack of autopsy confirmation of the diagnoses and the corresponding classification of the pathologies. We tried to overcome this limitation by placing more pressure on the CSF-based grouping of the subjects. Furthermore, the control group was recruited predominantly from close relatives of the AD patients hospitalized in the Center; in many instances, the control subjects expressed concerns regarding their cognitive functions, obviously associated with the every-day life experiences of living in the proximity of a closely related ill person (such as spouse or parent). This most probably clarifies why we observed decreased MMSE score in some of these subjects, which merely reflects their afflicted mood and mild anxiety. We also need to stress that the CSF-based categorization is not without limitations. Lumbar puncture is often regarded as an invasive procedure, and the NDD biomarkers characterize with high, but not one hundred percent, accuracy. Therefore, we believe that the diagnosis, and the classification of subjects in research studies, always requires a multi-expertise team including clinical neurochemist, neuropsychologist, neuroradiologist, and-last but not least—an experienced clinician. Certainly more studies are necessary to confirm our findings.

Acknowledgements The research leading to these results has received support from the Innovative Medicines Initiative Joint Undertaking under EMIF grant agreement $n^{\circ} 115372$, resources of which are composed of financial contribution from the European Union's Seventh Framework Programme (FP7/2007-2013) and EFPIA companies' in kind contribution. The study received financial support from Fundacja Alzheimerowska, Wrocław, Poland.

Data associated with this paper will be available upon a reasonable request.

Funding Open Access funding enabled and organized by Projekt DEAL.

\section{Declarations}

Conflict of interest PL received consultation and/or lecture honoraria from IBL International, Fujirebio Europe, AJ Roboscreen, and Roche.

Open Access This article is licensed under a Creative Commons Attribution 4.0 International License, which permits use, sharing, adaptation, distribution and reproduction in any medium or format, as long as you give appropriate credit to the original author(s) and the source, provide a link to the Creative Commons licence, and indicate if changes were made. The images or other third party material in this article are included in the article's Creative Commons licence, unless indicated otherwise in a credit line to the material. If material is not included in the article's Creative Commons licence and your intended use is not permitted by statutory regulation or exceeds the permitted use, you will need to obtain permission directly from the copyright holder. To view a copy of this licence, visit http://creativecommons.org/licenses/by/4.0/. 


\section{References}

Arai Y, Yamazaki M, Mori O, Muramatsu H, Asano G, Katayama Y (2001) Alpha-synuclein-positive structures in cases with sporadic Alzheimer's disease: morphology and its relationship to tau aggregation. Brain Res 888(2):287-296. https://doi.org/10.1016/ s0006-8993(00)03082-1

Baldeiras I, Santana I, Leitao MJ, Vieira D, Duro D, Mroczko B, Kornhuber J, Lewczuk P (2019) Erlangen score as a tool to predict progression from mild cognitive impairment to dementia in Alzheimer's disease. Alzheimers Res Ther 11(1):2. https://doi.org/ 10.1186/s13195-018-0456-x

Buddhala C, Campbell MC, Perlmutter JS, Kotzbauer PT (2015) Correlation between decreased CSF alpha-synuclein and Abeta(1) $(-)(4)(2)$ in Parkinson disease. Neurobiol Aging 36(1):476-484. https://doi.org/10.1016/j.neurobiolaging.2014.07.043

Burns JM, Galvin JE, Roe CM, Morris JC, McKeel DW (2005) The pathology of the substantia nigra in Alzheimer disease with extrapyramidal signs. Neurology 64(8):1397-1403. https://doi. org/10.1212/01.WNL.0000158423.05224.7F

Coughlin SS, Trock B, Criqui MH, Pickle LW, Browner D, Tefft MC (1992) The logistic modeling of sensitivity, specificity, and predictive value of a diagnostic test. J Clin Epidemiol 45(1):1-7

Ellis RJ, Caligiuri M, Galasko D, Thal LJ (1996) Extrapyramidal motor signs in clinically diagnosed Alzheimer disease. Alzheimer Dis Assoc Disord 10(2):103-114

Eusebi P, Giannandrea D, Biscetti L, Abraha I, Chiasserini D, Orso M, Calabresi P, Parnetti L (2016) Diagnostic utility of CSF alphasynuclein species in Parkinson's disease: protocol for a systematic review and meta-analysis. BMJ Open 6(6):e011113. https://doi. org/10.1136/bmjopen-2016-011113

Hamilton RL (2000) Lewy bodies in Alzheimer's disease: a neuropathological review of 145 cases using alpha-synuclein immunohistochemistry. Brain Pathol 10(3):378-384

Hansson O, Lehmann S, Otto M, Zetterberg H, Lewczuk P (2019) Advantages and disadvantages of the use of the CSF Amyloid beta (Abeta) 42/40 ratio in the diagnosis of Alzheimer's Disease. Alzheimers Res Ther 11(1):34. https://doi.org/10.1186/ s13195-019-0485-0

Korff A, Liu C, Ginghina C, Shi M, Zhang J, I Alzheimer's Disease Neuroimaging (2013) alpha-Synuclein in cerebrospinal fluid of Alzheimer's disease and mild cognitive impairment. J Alzheimers Dis 36(4):679-688. https://doi.org/10.3233/JAD-130458

Larson ME, Greimel SJ, Amar F, LaCroix M, Boyle G, Sherman MA, Schley H, Miel C, Schneider JA, Kayed R, Benfenati F, Lee MK, Bennett DA, Lesne SE (2017) Selective lowering of synapsins induced by oligomeric alpha-synuclein exacerbates memory deficits. Proc Natl Acad Sci U S A 114(23):E4648-E4657. https://doi. org/10.1073/pnas.1704698114

Lewczuk P, Kornhuber J, Toledo JB, Trojanowski JQ, Knapik-Czajka M, Peters O, Wiltfang J, Shaw LM (2015) Validation of the Erlangen score algorithm for the prediction of the development of dementia due to Alzheimer's disease in pre-dementia subjects. J Alzheimers Dis 49(3):887. https://doi.org/10.3233/JAD-159006

Lewczuk P, Riederer P, Oryant SE, Verbeek MM, Dubois B, Visser PJ, Jellinger KA, Engelborghs S, Ramirez A, Parnetti L, Jack CR Jr, Teunissen CE, Hampel H, Lleo A, Jessen F, Glodzik L, de Leon MJ, Fagan AM, Molinuevo JL, Jansen WJ, Winblad B, Shaw LM, Andreasson U, Otto M, Mollenhauer B, Wiltfang J, Turner MR, Zerr I, Handels R, Thompson AG, Johansson G, Ermann N, Trojanowski JQ, Karaca I, Wagner H, Oeckl P, van Waalwijk DL, Bjerke M, Kapogiannis D, Kuiperij HB, Farotti L, Li Y, Gordon BA, Epelbaum S, Vos SJB, Klijn CJM, Van Nostrand WE, Minguillon C, Schmitz M, Gallo C, Lopez Mato A, Thibaut F, Lista S, Alcolea D, Zetterberg H, Blennow K, Kornhuber J, Members of the Wfsbp Task Force Working on this Topic: Peter Riederer CGDKALMFT (2018) Cerebrospinal fluid and blood biomarkers for neurodegenerative dementias: an update of the consensus of the task force on biological markers in psychiatry of the world federation of societies of biological psychiatry. World J Biol Psychiatry 19(4):244-328. https://doi.org/10.1080/15622975.2017. 1375556

Lewczuk P, Zimmermann R, Wiltfang J, Kornhuber J (2009) Neurochemical dementia diagnostics: a simple algorithm for interpretation of the CSF biomarkers. J Neural Transm 116(9):1163-1167. https://doi.org/10.1007/s00702-009-0277-y

Lippa CF, Fujiwara H, Mann DM, Giasson B, Baba M, Schmidt ML, Nee LE, O'Connell B, Pollen DA, St George-Hyslop P, Ghetti B, Nochlin D, Bird TD, Cairns NJ, Lee VM, Iwatsubo T, Trojanowski JQ (1998) Lewy bodies contain altered alpha-synuclein in brains of many familial Alzheimer's disease patients with mutations in presenilin and amyloid precursor protein genes. Am J Pathol 153(5):1365-1370. https://doi.org/10.1016/ s0002-9440(10)65722-7

Liu Y, Stern Y, Chun MR, Jacobs DM, Yau P, Goldman JE (1997) Pathological correlates of extrapyramidal signs in Alzheimer's disease. Ann Neurol 41(3):368-374. https://doi.org/10.1002/ ana.410410312

Mackin RS, Insel P, Zhang J, Mohlenhoff B, Galasko D, Weiner M, Mattsson N (2015) Cerebrospinal fluid alpha-synuclein and Lewy body-like symptoms in normal controls, mild cognitive impairment, and Alzheimer's disease. J Alzheimers Dis 43(3):1007-1016. https://doi.org/10.3233/JAD-141287

Majbour NK, Chiasserini D, Vaikath NN, Eusebi P, Tokuda T, van de Berg W, Parnetti L, Calabresi P, El-Agnaf OM (2017) Increased levels of CSF total but not oligomeric or phosphorylated forms of alpha-synuclein in patients diagnosed with probable Alzheimer's disease. Sci Rep 7:40263. https://doi.org/10.1038/srep4 0263

Marui W, Iseki E, Ueda K, Kosaka K (2000) Occurrence of human alpha-synuclein immunoreactive neurons with neurofibrillary tangle formation in the limbic areas of patients with Alzheimer's disease. J Neurol Sci 174(2):81-84

McKhann GM, Knopman DS, Chertkow H, Hyman BT, Jack CR Jr, Kawas CH, Klunk WE, Koroshetz WJ, Manly JJ, Mayeux R, Mohs RC, Morris JC, Rossor MN, Scheltens P, Carrillo MC, Thies B, Weintraub S, Phelps CH (2011) The diagnosis of dementia due to Alzheimer's disease: recommendations from the National Institute on Aging-Alzheimer's Association workgroups on diagnostic guidelines for Alzheimer's disease. Alzheimers Dement 7(3):263-269

Mollenhauer B, Cullen V, Kahn I, Krastins B, Outeiro TF, Pepivani I, Ng J, Schulz-Schaeffer W, Kretzschmar HA, McLean PJ, Trenkwalder C, Sarracino DA, Vonsattel JP, Locascio JJ, El-Agnaf OM, Schlossmacher MG (2008) Direct quantification of CSF alphasynuclein by ELISA and first cross-sectional study in patients with neurodegeneration. Exp Neurol 213(2):315-325. https://doi.org/ 10.1016/j.expneurol.2008.06.004

Oeckl P, Metzger F, Nagl M, von Arnim CA, Halbgebauer S, Steinacker P, Ludolph AC, Otto M (2016) Alpha-, Beta-, and gammasynuclein quantification in cerebrospinal fluid by multiple reaction monitoring reveals increased concentrations in Alzheimer's and creutzfeldt-jakob disease but no alteration in synucleinopathies. MCP 15(10):3126-3138. https://doi.org/10.1074/mcp.M116. 059915

Parnetti L, Cicognola C, Eusebi P, Chiasserini D (2016) Value of cerebrospinal fluid alpha-synuclein species as biomarker in Parkinson's diagnosis and prognosis. Biomark Med 10(1):35-49. https:// doi.org/10.2217/bmm.15.107

Portet F, Scarmeas N, Cosentino S, Helzner EP, Stern Y (2009) Extrapyramidal signs before and after diagnosis of incident 
Alzheimer disease in a prospective population study. Arch Neurol 66(9):1120-1126. https://doi.org/10.1001/archneurol.2009.196

Scheltens P, Leys D, Barkhof F, Huglo D, Weinstein HC, Vermersch P, Kuiper M, Steinling M, Wolters EC, Valk J (1992) Atrophy of medial temporal lobes on MRI in "probable" Alzheimer's disease and normal ageing: diagnostic value and neuropsychological correlates. J Neurol Neurosurg Psychiatry 55(10):967-972. https:// doi.org/10.1136/jnnp.55.10.967

Simonsen AH, Kuiperij B, El-Agnaf OM, Engelborghs S, Herukka SK, Parnetti L, Rektorova I, Vanmechelen E, Kapaki E, Verbeek M, Mollenhauer B (2016) The utility of alpha-synuclein as biofluid marker in neurodegenerative diseases: a systematic review of the literature. Biomark Med 10(1):19-34. https://doi.org/10.2217/ BMM.14.105

Skillback T, Kornhuber J, Blennow K, Zetterberg H, Lewczuk P, Alzheimer's Disease Neuroimaging I (2019) Erlangen score predicts cognitive and neuroimaging progression in mild cognitive impairment stage of alzheimer's disease. J Alzheimers Dis 69(2):551559. https://doi.org/10.3233/JAD-190067

Slaets S, Le Bastard N, Martin JJ, Sleegers K, Van Broeckhoven C, De Deyn PP, Engelborghs S (2013) Cerebrospinal fluid Abeta1-40 improves differential dementia diagnosis in patients with intermediate P-tau181P levels. J Alzheimers Dis 36(4):759-767

Slaets S, Vanmechelen E, Le Bastard N, Decraemer H, Vandijck M, Martin JJ, De Deyn PP, Engelborghs S (2014) Increased CSF alpha-synuclein levels in Alzheimer's disease: correlation with tau levels. Alzheimers Dement 10(5 Suppl):S290-298. https://doi. org/10.1016/j.jalz.2013.10.004

Somers C, Lewczuk P, Sieben A, Van Broeckhoven C, De Deyn PP, Kornhuber J, Martin JJ, Bjerke M, Engelborghs S (2019) Validation of the Erlangen score algorithm for differential dementia diagnosis in autopsy-confirmed subjects. J Alzheimers Dis 68(3):1151-1159. https://doi.org/10.3233/JAD-180563

Toledo JB, Cairns NJ, Da X, Chen K, Carter D, Fleisher A, Householder E, Ayutyanont N, Roontiva A, Bauer RJ, Eisen P, Shaw LM, Davatzikos C, Weiner MW, Reiman EM, Morris JC, Trojanowski JQ, Alzheimer's Disease Neuroimaging I (2013) Clinical and multimodal biomarker correlates of ADNI neuropathological findings. Acta Neuropathol Commun 1:65. https://doi.org/10. 1186/2051-5960-1-65

Twohig D, Nielsen HM (2019) alpha-synuclein in the pathophysiology of Alzheimer's disease. Mol Neurodegener 14(1):23. https://doi. org/10.1186/s13024-019-0320-x

Publisher's Note Springer Nature remains neutral with regard to jurisdictional claims in published maps and institutional affiliations. 